\title{
Hairiness: the missing link between pollinators and pollination
}

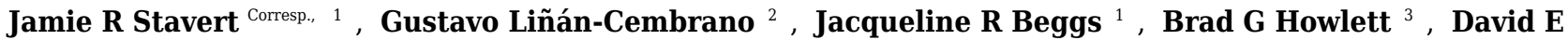 \\ Pattemore $^{4}$, Ignasi Bartomeus ${ }^{5}$ \\ ${ }^{1}$ Centre for Biodiversity and Biosecurity, School of Biological Sciences, The University of Auckland, Auckland, New Zealand \\ 2 Instituto de Microelectrónica de Sevilla CSIC / Universidad de Sevilla, Sevilla, Spain \\ 3 The New Zealand Institute for Plant \& Food Research Limited, Christchurch, New Zealand \\ 4 The New Zealand Institute for Plant \& Food Research Limited, Hamilton, New Zealand \\ Integrative Ecology Department, Estación Biológica de Doñana (EBD-CSIC), Sevilla, Spain \\ Corresponding Author: Jamie R Stavert \\ Email address: jamie.stavert@gmail.com
}

Background. Functional traits are the primary biotic component driving organism influence on ecosystem functions; in consequence, traits are widely used in ecological research. However, most animal trait-based studies use easy-to-measure characteristics of species that are at best only weakly associated with functions. Animal-mediated pollination is a key ecosystem function and is likely to be influenced by pollinator traits, but to date no one has identified functional traits that are simple to measure and have good predictive power.

Methods. Here, we show that a simple, easy to measure trait (hairiness) can predict pollinator effectiveness with high accuracy. We used a novel image analysis method to calculate entropy values for insect body surfaces as a measure of hairiness. We evaluated the power of our method for predicting pollinator effectiveness by regressing pollinator hairiness (entropy) against single visit pollen deposition (SVD) and pollen loads on insects. We used linear models and $\mathrm{AlC}_{\mathrm{C}}$ model selection to determine which body regions were the best predictors of SVD and pollen load.

Results. We found that hairiness can be used as a robust proxy of SVD. The best models for predicting SVD for the flower species Brassica rapa and Actinidia deliciosa were hairiness on the face and thorax as predictors ( $R^{2}=0.98$ and 0.91 respectively). The best model for predicting pollen load for $B$. rapa was hairiness on the face $\left(R^{2}=0.81\right)$.

Discussion. We suggest that the match between pollinator body region hairiness and plant reproductive structure morphology is a powerful predictor of pollinator effectiveness. We show that pollinator hairiness is strongly linked to pollination - an important ecosystem function, and provide a rigorous and timeefficient method for measuring hairiness. Identifying and accurately measuring key traits that drive ecosystem processes is critical as global change increasingly alters ecological communities, and subsequently, ecosystem functions worldwide. 
1 Title

2 Hairiness: the missing link between pollinators and pollination

3

4 Authors

5 Jamie R. Stavert ${ }^{1 *}$, Gustavo Liñán ${ }^{2}$, Jacqueline R. Beggs ${ }^{1}$, Brad G. Howlett ${ }^{3}$, David E.

6 Pattemore $^{4}$, and Ignasi Bartomeus ${ }^{5}$

7

8 *Corresponding author: jamie.stavert@gmail.com

9 Corresponding author ORCID ID: 0000-0002-2103-5320

$10{ }^{1}$ Centre for Biodiversity and Biosecurity, School of Biological Sciences, The University of

11 Auckland, Auckland, New Zealand

$12{ }^{2}$ Instituto de Microelectrónica de Sevilla (IMSE-CNM), Avda. Américo Vespucio s/n, Isla de la

13 Cartuja, E-41092 Sevilla, Spain

$14{ }^{3}$ The New Zealand Institute for Plant \& Food Research Limited, Christchurch, New Zealand

$15^{4}$ The New Zealand Institute for Plant \& Food Research Limited, Hamilton, New Zealand

$16{ }^{5}$ Estación Biológica de Doñana (EBD-CSIC), Integrative Ecology Department. Avda. Américo

17 Vespucio s/n, Isla de la Cartuja, E-41092 Sevilla, Spain 
19 Abstract

20

21 Background. Functional traits are the primary biotic component driving organism influence on

22 ecosystem functions; in consequence traits are widely used in ecological research. However,

23 most animal trait-based studies use easy-to-measure characteristics of species that are at best

24 only weakly associated with functions. Animal-mediated pollination is a key ecosystem function

25 and is likely to be influenced by pollinator traits, but to date no one has identified functional

26 traits that are simple to measure and have good predictive power.

27

28 Methods. Here, we show that a simple, easy to measure trait (hairiness) can predict pollinator

29 effectiveness with high accuracy. We used a novel image analysis method to calculate entropy

30 values for insect body surfaces as a measure of hairiness. We evaluated the power of our method

31 for predicting pollinator effectiveness by regressing pollinator hairiness (entropy) against single

32 visit pollen deposition (SVD) and pollen loads on insects. We used linear models and $\mathrm{AIC}_{\mathrm{C}}$

33 model selection to determine which body regions were the best predictors of SVD and pollen

34 load.

35

36 Results. We found that hairiness can be used as a robust proxy of SVD. The best models for

37 predicting SVD for the flower species Brassica rapa and Actinidia deliciosa were hairiness on

38 the face and thorax as predictors $\left(\mathrm{R}^{2}=0.98\right.$ and 0.91 respectively). The best model for predicting

39 pollen load for B. rapa was hairiness on the face $\left(\mathrm{R}^{2}=0.81\right)$.

40 
41 Discussion. We suggest that the match between pollinator body region hairiness and plant

42 reproductive structure morphology is a powerful predictor of pollinator effectiveness. We show

43 that pollinator hairiness is strongly linked to pollination - an important ecosystem function, and

44 provide a rigorous and time-efficient method for measuring hairiness. Identifying and accurately

45 measuring key traits that drive ecosystem processes is critical as global change increasingly

46 alters ecological communities, and subsequently, ecosystem functions worldwide.

47

\section{Introduction}

49 Trait-based approaches are now widely used in functional ecology, from the level of individual 50 organisms to ecosystems (Cadotte et al. 2011). Functional traits are defined as the characteristics

51 of an organism's phenotype that determine its effect on ecosystem level processes (Naeem \&

52 Wright 2003; Petchey \& Gaston 2006). Accordingly, functional traits are recognised as the

53 primary biotic component by which organisms influence ecosystem functions (Gagic et al. 2015;

54 Hillebrand \& Matthiessen 2009). Trait-based research is dominated by studies on plants and

55 primary productivity, and little is known about key traits for animal-mediated and multi-trophic

56 functions, particularly for terrestrial invertebrates (Didham et al. 2016; Gagic et al. 2015; Lavorel

57 et al. 2013).

58

59 Most animal trait-based studies simply quantify easy-to-measure morphological characteristics, 60 without a mechanistic underpinning to demonstrate these "traits" have any influence on the 61 ecosystem function of interest (Didham et al. 2016). This results in low predictive power,

62 particularly where trait selection lacks strong justification through explicit ecological questions

63 (Gagic et al. 2015; Petchey \& Gaston 2006). If the ultimate goal of trait-based ecology is to 
64 identify the mechanisms that drive biodiversity impacts on ecosystem function, then traits must

65 be quantifiable at the level of the individual organism, and be inherently linked to an ecosystem

66 function (Bolnick et al. 2011; Pasari et al. 2013; Violle et al. 2007).

67

68 Methodology that allows collection of trait data in a rigorous yet time-efficient manner and with 69 direct functional interpretation will greatly enhance the power of trait-based studies. Instead of 70 subjectively selecting a large number of traits with unspecified links to ecosystem functions, it

71 would be better to identify fewer, uncorrelated traits, that have a strong bearing on the function

72 of interest (Carmona et al. 2016). Selecting traits that are measurable on a continuous scale,

73 would also improve predictive power of studies (McGill et al. 2006; Violle et al. 2012).

74 However, far greater time and effort is required to measure such traits, exacerbating the already 75 demanding nature of trait-based community ecology (Petchey \& Gaston 2006).

76

77 Animal-mediated pollination is a multi-trophic function, driven by the interaction between

78 animal pollinators and plants (Kremen et al. 2007). A majority of the world's wild plant species

79 are pollinated by animals (Ollerton et al. 2011), and over a third of global crops are dependent on

80 animal pollination (Klein et al. 2007b). Understanding which pollinator traits determine the

81 effectiveness of different pollinators is critical to understanding the mechanisms of pollination

82 processes. However, current traits used in pollination studies often have weak associations with

83 pollination function and/or have low predictive power. For example Larsen, Williams \& Kremen

84 (2005) used body mass to explain pollen deposition by solitary bees even when the relationship

85 was weak and non-significant. Many trait-based pollination studies have subsequently used body

86 mass or similar size measures, despite their low predictive power. Similarly, Hoehn et al (2008) 
87 used spatial and temporal visitation preferences of bees to explain differences in plants

88 reproductive output. They found significant relationships (i.e. low $P$ values) between spatial and

89 temporal visitation preferences and seed set, but with small $\mathrm{R}^{2}$ values, suggesting these traits

90 have weak predictive power. To advance trait-based pollination research we require traits that are

91 good predictors of pollination success.

92

93 Observational studies suggest that insect body hairs are important for collecting pollen that is

94 used by insects for food and larval provisioning (Holloway 1976; Thorp 2000). Hairs facilitate

95 active pollen collection, e.g., many bees have specialised hair structures called scopae that are

96 used to transport pollen to the nest for larval provisioning (Thorp 2000). Additionally, both bees

97 and flies have hairs distributed across their body surfaces which act to passively collect pollen

98 for adult feeding (Holloway 1976). Differences in the density and distribution of hairs on pollen

99 feeding insects likely reflects their feeding behaviour, the types of flowers they visit, and

100 whether they use pollen for adult feeding and/or larval provisioning (Thorp 2000). However,

101 despite anecdotal evidence that insect body hairs are important for pollen collection and

102 pollination, there is no proven method for measuring hairiness, nor is there evidence that hairier

103 insects are more effective pollinators.

104

105 Here, we present a novel method based on image entropy analysis for quantifying pollinator

106 hairiness. We define pollination effectiveness as single visit pollen deposition (SVD): the

107 number of conspecific pollen grains deposited on a virgin stigma in a single visit (King et al.

108 2013; Ne'eman et al. 2010). SVD is a measure of an insects' ability to acquire free pollen grains

109 on the body surface and accurately deposit them on a conspecific stigma. We predict that 
110 hairiness, specifically on the body parts that contact the stigma, will have a strong association

111 with SVD. We show that the best model for predicting pollinator SVD for pak choi Brassica

112 rapa is highly predictive and includes hairiness of the face and thorax dorsal regions as

113 predictors, and the face region alone explains more than $90 \%$ of the variation. Similarly, the best

114 model for predicting SVD for kiwifruit Actinidia deliciosa includes the face and thorax ventral

115 regions and has good predictive power. Our novel method for measuring hairiness is rigorous,

116 time efficient and inherently linked to pollination function. Accordingly, this method could be

117 applied in diverse trait-based pollination studies to progress understanding of the mechanisms

118 that drive pollination processes.

119

120 Materials and Methods

121 Imaging for hairiness analysis

122 We photographed pinned insect specimens using the Visionary Digital Passport portable imaging

123 system (Figure 1). Images were taken with a Canon EOS 5D Mark II digital camera (5616 x

1243744 pix). The camera colour profile was sRGB IEC61966-2.1, focal length was 65mm and F-

125 number was 4.5. We used ventral, dorsal and frontal shots with clear illumination to minimise

126 reflection from shinny insect body surfaces. All photographs were taken on a plain white

127 background. Raw images were exported to Helicon Focus 6 where they were stacked and stored 128 in .jpg file format.

129

\section{Image processing and analysis}

131 We produced code to quantify insect pollinator hairiness using MATLAB (MathWorks, Natick, 132 MA, USA), and functions from the MATLAB Image Processing ToolBox. We quantified 
133 relative hairiness by creating an entropy image for each insect photograph, and computed the

134 average entropy within user-defined regions (Gonzales et al. 2004). To calculate entropy values

135 for each image we designed three main functions. The first function allows the user to define up

136 to four regions of interest (RoIs) within each image. The user can define regions by drawing

137 contours as closed polygonal lines of any arbitrary number of vertexes. All information about

138 regions (location, area and input image file name) is stored as a structure in a .mat file.

139

140 The second function executes image pre-processing. We found that some insects had pollen

141 grains or other artefacts attached to their bodies, which would alter the entropy results. Our pre-

142 processing function eliminates these objects from the image by running two filtering processes.

143 First, the function eliminates small objects with an area less than the user definable threshold (8

144 pixels by default). For the first task, each marked region is segmented using an optimized

145 threshold obtained by applying a spatially dependant thresholding technique. Once each region

146 has been segmented, a labelling process is executed for all resulting objects and those with an

147 area smaller than the minimum value defined by the user are removed. Secondly, as pollen grains

148 are often round in shape, the function eliminates near-circular objects. The perimeter of each

149 object is calculated and its similarity to a circle $(\mathrm{S})$ id defined as:

150

$$
S=\frac{4 \pi \cdot \text { Area }^{2}}{\text { Perimeter }^{2}}
$$

151 Objects with a similarity coefficient not within the bounds defined by the user (5\% by default)

152 are also removed from the image. Perimeter calculation is carried out by finding the object's

153 boundary, and computing the accumulated distance from pixel centre to pixel centre across the

154 border, rather than simply counting the number of pixels in the border. The entropy filter will not

155 process objects that have been marked as "deleted" by the pre-processing function. This initial 
156 pre-processing provides flexibility by allowing users to define the minimum area threshold and

157 the degree of similarity of objects to a circle. Users can also disable the image pre-processing by

158 toggling a flag when running the entropy filter.

159

160 Once pre-processing is complete, each image is passed to the third function, which is the entropy

161 filter calculation stage. The entropy filter produces an overall measure of randomness within

162 each of the user defined regions on the image. In information theory, entropy (also expressed as

163 Shannon Entropy) is an indicator of the average amount of information contained in a message

164 (Shannon 1948). Therefore, Shannon Entropy, H, of a discrete random variable $X$ that can take $n$

165 possible values $\left\{x_{1}, x_{2}, \ldots, x_{n}\right\}$, with a probability mass function $P(X)$ is given by:

166

$$
H(X)=-\sum_{i=1}^{n} P\left(x_{i}\right) \cdot \log _{2}\left(P\left(x_{i}\right)\right)
$$

167 When this definition is used in image processing, local entropy defines the degree of complexity

168 (variability) within a given neighbourhood around a pixel. In our case, this neighbourhood (often

169 referred to as the structuring element) is a disk with radius $r$ (we call the radius of influence) that

170 can be defined by the user ( 7 pixels by default). Thus for a given pixel in position $(i, j)$ in the input

171 image, the entropy filter computes the histogram $G_{i j}$ (using 256 bins) of all pixels within its radius

172 of influence, and returns its entropy value $H_{i j}$ as:

173

$$
H_{i j}=-G_{i j} \cdot \log _{2}\left(G_{i j}\right)
$$

174 where $G_{i j}$ is a vector containing the histogram results for pixel $(i, j)$ and $(\cdot)$ is the dot product

175 operator. Using default parameters, our entropy filter employs a 7 pixel (13 x 13 neighbourhood)

176 radius of influence, and a disk-shaped structuring element, which we determined based on the

177 size of hairs. Therefore, in the entropy image, each pixel takes a value of entropy when 
178 considering 160 pixels around it (by default). We determined the optimal radius of influence for

179 the entropy filter by running our entropy function with the radius of influence set as a variable

180 parameter. We then visually compared the contrast in areas of low vs. high hairiness in the

181 resulting entropy images (i.e. Figure 1). We found that a 7 pixel radius of influence gave the best

182 contrast between low and high hairiness areas for our species set. Hair thickness values across

183 species typically ranged between $3.5-4.5$ pixels and therefore, the 7 pixel radius of influence is

184 approximately two times the width of a hair.

185

186 The definition of the optimum radius of influence depends on the size of the morphological

187 responsible for the complexity in the RoI. This is defined not only by the physical size of these

188 features but also by the pixel-to-millimetre scaling factor (i.e. number of pixels in the sensor

189 plane per $\mathrm{mm}$ in the scene plane). Thus, although 7 pixels is the optimum in our case to detect

190 hairs, the entropy filter function takes this radius as an external parameter which can be adjusted

191 by the user to meet their needs.

192

193 The entropy filter function is a process that runs over three different entropy layers $\left(E_{R}, E_{G}, E_{B}\right)$,

194 one for each of the camera's colour channels (Red, Green, and Blue), for each input image.

195 These three images are combined into a final combined entropy image $\mathrm{E}_{\mathrm{S}}$, where each pixel in 196 position $(i, j)$ takes the value $\mathrm{E}_{\mathrm{S}(i, j)}$ :

$$
E_{S(i, j)}=E_{R(i, j)} \cdot E_{G(i, j)} \cdot E_{G(i, j)}
$$

198 Once entropy calculations are complete, our function computes averages and standard deviations 199 of $E_{S}$ within each of the regions previously defined by the user, and writes the results into a .csv 200 file (one row per image). Entropy values produced by this function are consistent for different 
201 photos of the same region on the same specimen (Supporting Information 6; Table S2). The

202 scripts for the image pre-processing, region marking and entropy analysis functions are provided,

203 along with a MATLAB tutorial (Supporting Information 1-4).

204

205

Hairiness as a predictor of SVD and pollen load

206

207 Model flower floral biology and pollinator collection

208 We used pak choi Brassica rapa var. chinensis (Brassicaceae) and kiwifruit Actinidia deliciosa

209 (Actinidiaceae) as model flowers to determine if our measurement of insect hairiness is a good

210 predictor of pollinator effectiveness.

211

212 Both B. rapa and A. deliciosa are important mass flowering global food crops (Klein et al.

213 2007a; Rader et al. 2009). B. rapa has an actinomorphic open pollinated yellow flower with four

214 sepals, four petals, and six stamens (four long and two short) (Walker et al. 1999). The nectaries

215 are located in the centre of the flower, between the stamens and the petals, forcing pollinators to

216 introduce their head between the petals. B. rapa shows increased seed set in the presence of

217 insect pollinators and the flowers are visited by a diverse assemblage of insects that differ in their

218 ability to transfer pollen (Rader et al. 2013). A. deliciosa is dioecious with individual plants

219 producing either male or female flowers. Flowers are large (4-6 cm in diameter) and typically

220 have 5-9 white/cream coloured petals (Devi et al. 2015). Flowers have multiple stamens and

221 staminodes with yellow anthers. Female flowers have a large stigma with multiple branches that

222 form a brush-like structure. Both male and female flowers do not produce nectar but both

223 produce pollen, which acts as a reward to visitors. Like B. rapa, A. deliciosa flowers are visited 
224 by a diverse range of insects that differ in their ability to transfer pollen, and seed set is increased

225 in the presence of insect pollinators (Craig et al. 1988).

226

227 We collected pollinating insects for image analysis during the summer of December 2014 -

228 January 2015. Insects were chilled immediately and then killed by freezing within 1 day and 229 stored at $-18^{\circ} \mathrm{C}$ in individual vials. All insects were identified to species level with assistance 230 from expert taxonomists.

231

\section{Image processing}

233 We measured the hairiness of 10 insect pollinator species ( $n=8-10$ individuals per species),

234 across five families and two orders. This included social, semi-social and solitary bees and 235 pollinating flies. Regions marked included: 1) face; 2) head dorsal; 3) head ventral; 4) front leg;

236 5) thorax dorsal; 6) thorax ventral; 7) abdomen dorsal and 8) abdomen ventral. All entropy

237 analysis was carried out using our image processing method outlined above. For estimates of

238 body size, we took multiple linear measurements (body length, body width, head length, head

239 width, foreleg length and hind leg length) of each specimen using digital callipers and a

240 dissecting microscope.

241

242 Single visit pollen deposition (SVD) and pollen load

243 For B. rapa we used SVD data for insect pollinators presented in Rader et al. (2009) and Howlett

244 et al. (2011); a brief description of their methods follows.

245 
246 Pollen deposition on stigmatic surfaces (SVD) was estimated using manipulation experiments.

247 Virgin B. rapa inflorescences were bagged to exclude all pollinators. Once flowers had opened,

248 the bag was removed, and flowers were observed until an insect visited and contacted the stigma

249 in a single visit. The stigma was then removed and stored in gelatine-fuchsin and the insect was

250 captured for later identification. SVD was quantified by counting all B. rapa pollen grains on the

251 stigma. Mean values of SVD for each species are used in our regression models.

252

253 To quantify the number of pollen grains carried (pollen load), sensu Howlett et al. (2011),

254 collected insects while foraging on B. rapa flowers. Insects were captured using plastic vials

255 containing a rapid killing agent (ethyl acetate). Once dead, a cube of gelatine-fuchsin was used to

256 remove all pollen from the insect's body surface. Pollen collecting structures (e.g., corbiculae,

257 scopae) were not included in analyses because pollen from these structures is not available for

258 pollination. Slides were prepared in the field by melting the gelatine-fuchsin cubes containing

259 pollen samples onto microscope slides. B. rapa pollen grains from each sample were then

260 quantified by counting pollen grains in an equal-area subset from the sample and multiplying this

261 by the number of equivalent sized subset areas within the total sample.

262

263 We measured SVD for $A$. deliciosa ( $n=8-12$ per pollinator species). SVD measurements were

264 taken for insect movements from staminate to pistillate flowers, using a method that differed

265 from B. rapa. Individual pistillate buds were enclosed within paper bags 2-3 days prior to

266 opening, and were later used as test flowers to evaluate pollen deposition by flowering visiting

267 species. Each bag was secured using a wire tie (coated in plastic) that was gently twisted to

268 exclude pollinators from visiting the opening flowers. Following flower opening, the bag was 
269 removed and the flower pedicel abscised where it joined the vine. The test flower was then

270 carefully positioned using forceps to hold the pedicel $1-2 \mathrm{~cm}$ from a staminate flower containing

271 a foraging insect, avoiding any contacting between flowers. If the test flower was visited by an

272 insect, we allowed it to forage with minimal disturbance until it moved from the flower on its

273 own accord. The first stigma touched by the foraging insect was then lightly marked near its base

274 using a fine black felt pen. We then placed the marked stigma onto a slide and applied a drop of

275 Alexander stain (Dafni 2007). Alexander stain was used due to its effectiveness to stain

276 staminate and pistillate pollen differently (pistillate pollen - green-blue, staminate pollen - dark

277 red) (Goodwin \& Perry 1992).

278

279 Statistical analyses

280 We used linear regression models and $\mathrm{AIC}_{\mathrm{C}}$ (small sample corrected Akaike information criteria)

281 model selection to determine if our measure of pollinator hairiness is a good predictor of SVD

282 and pollen load. We constructed global models with SVD or pollen load as the response variable,

283 body region as predictors and body length as an interaction i.e. SVD or pollen load body length

$284 *$ entropy face + entropy head dorsal + entropy head ventral + front leg + entropy thorax dorsal +

285 entropy thorax ventral + entropy abdomen dorsal + entropy abdomen ventral. We included body

286 length in our global model as a proxy for body size as it had high correlation coefficients

287 (Pearson's $r>0.7$ ) with all other body size measurements. Global linear models were constructed

288 using the $\mathrm{lm}$ (stats) function. $\mathrm{AIC}_{\mathrm{C}}$ model selection was carried out on the global models using

289 the function glmulti() with fitfunction $=$ "lm" in the package glmulti. We examined

290 heteroscedasticity and normality of errors of models by visually inspecting diagnostic plots using

291 the glmulti package (Crawley 2002). Variance inflation factors (VIF) of predictor variables were 
292 checked for the best models using the vif() function in the car package. All analyses were done

293 in R version 3.2.4 (R Development Core Team 2014).

294

295 Results

296 Body hairiness as a predictor of SVD

297 For SVD on B. rapa, the face and thorax dorsal regions were retained in the best model selected 298 by $\mathrm{AIC}_{\mathrm{C}}$, which had an adjusted $\mathrm{R}^{2}$ value of 0.98 . The subsequent top models within $10 \mathrm{AIC}_{\mathrm{C}}$ 299 points all retained the face and thorax dorsal regions and additionally included the abdomen 300 ventral (adjusted $\mathrm{R}^{2}=0.98$ ), head dorsal (adjusted $\mathrm{R}^{2}=0.98$ ), and thorax ventral (adjusted $\mathrm{R}^{2}=$ 3010.97 ) and front leg (adjusted $\mathrm{R}^{2}=0.97$ ) regions respectively (Table 1; Figure 2). The model with 302 the face region included as a single predictor had an adjusted $\mathrm{R}^{2}$ value of 0.88 , indicating that this 303 region alone explained a majority of the variation in the top SVD models.

304

305 The best model for predicting SVD on A. deliciosa included the face and thorax ventral regions 306 as predictors (adjusted $\mathrm{R}^{2}=0.91$ ) (Table 1; Figure 3). However, the subsequent top four models

307 were within two $\mathrm{AIC}_{\mathrm{C}}$ points of the best model and therefore cannot be discounted as the 308 potential top model. The face, thorax ventral, head ventral and abdomen ventral regions were 309 retained in four of the five top models, which indicates that hairiness of the face and ventral 310 regions is important for pollen deposition on A. deliciosa. For both B. rapa and A. deliciosa, 311 body length and the body length interaction were not included in the top models.

312

313 Body hairiness as a predictor of pollen load 
314 The best model for pollen load retained the face region only and had an adjusted $\mathrm{R}^{2}$ value of 0.81

315 (Figure 4; Table 1). The subsequent best models retained the abdomen dorsal (adjusted $\mathrm{R}^{2}$ value

316 of 0.73 ), the face and head dorsal (adjusted $\mathrm{R}^{2}=0.83$ ), the face and abdomen dorsal (adjusted $\mathrm{R}^{2}$

$317=0.82)$ and the abdomen dorsal and front leg (adjusted $\mathrm{R}^{2}=0.8$ ) regions respectively. For pollen

318 load, body length and the body length interaction were not included in the top models.

320 Discussion

321 Here we present a rigorous and time-efficient method for quantifying hairiness, and demonstrate

322 that this measure is an important pollinator functional trait. We show that insect pollinator

323 hairiness is a strong predictor of SVD for the open-pollinated flower B. rapa. Linear models that

324 included multiple body regions as predictors had the highest predictive power; the top model for

325 SVD retained the face and thorax dorsal regions. However, the face region was retained in all of

326 the top models, and when included as a single predictor, had a very strong positive association

327 with SVD. In addition, we show that hairiness, particularly on the face and ventral regions, is a

328 good predictor of SVD for A. deliciosa, which has a different floral morphology, suggesting our

329 method could be suitable for a range of flower types. Hairiness was also a good predictor for

330 pollen load, and the face region was again retained in the top model for B. rapa. The abdomen

331 dorsal, head dorsal and front leg regions were also good predictors of pollen load and were

332 retained in the subsequent top models. Our results validate the importance of insect body hairs

333 for transporting and depositing pollen. Surprisingly, we did not find strong associations between

334 SVD and body size, and top models did not contain the body length interaction. Similarly, body

335 length was not retained in the top models for pollen load. This indicates that our measure of

336 hairiness has far greater predictive power than body size for both SVD and pollen load. 
338 When deciding on which body regions to measure hairiness, researchers may first need to assess

339 additional pollinator traits, such as flower visiting behaviour. This is because the way in which

340 insects interact with flowers influences what body parts most frequently contact the floral

341 reproductive structures (Roubik 2000). For some open pollinated flowers, such as $B$. rapa, facial

342 hairs are probably the most important for pollen deposition because the face is the most likely

343 region to contact the anthers and stigma. However, for flowers with different floral

344 morphologies, facial hairs may not be as important because the floral reproductive structures

345 have different positions relative to the insect's body structures. For example, disc-shaped flowers

346 tend to deposit their pollen on the ventral regions of pollinators, while labiate flowers deposit

347 their pollen on the dorsal regions (Bartomeus et al. 2008). We found that hairiness on the face

348 and ventral regions of pollinators was most important for pollen deposition on $A$. deliciosa

349 flowers. The reproductive parts of $A$. deliciosa form a brush shaped structure and therefore are

350 most likely to contact the face and ventral surfaces of pollinators. Accordingly, where studies

351 focus on a single plant species i.e. crop based studies, it is important to consider trait matching

352 when selecting pollinator body region(s) to analyse (Butterfield \& Suding 2013; Garibaldi et al.

353 2015).

354

355 It is important to consider that pollinator performance is a function of both SVD and visitation

356 frequency, and these two components operate independently (Kremen et al. 2002; Mayfield et al.

357 2001). Here, we focus on a single trait that is important for pollinator efficiency (SVD), but to

358 calculate pollinator performance researchers need to measure both efficiency and visitation rate.

359 Additional pollinator traits related to visitation rate, as well as other behavioural traits such as 
360 activity patterns relative to the timing of stigma receptivity (Potts et al. 2001) and foraging

361 behaviour, e.g., nectar vs. pollen foraging (Herrera 1987; Javorek et al. 2002; Rathcke 1983),

362 may be important for predicting pollination performance. In some circumstances it might also be

363 important to consider trait differences between male and female pollinators, particularly for some

364 bee species. Male and female bees may have different pollen deposition efficiency due to

365 differences in their foraging behaviour and resource requirements. For example, female bees are

366 likely to visit flowers to collect pollen for nest provisioning while males simply consume nectar

367 and pollen during visits (Cane et al. 2011). For some flowers, male bees have a similar

368 pollination efficiency compared to females (e.g., summer squash Cucurbita pepo; Cane et al.

369 2011) while for others, female bees are more effective than males (e.g., lowbush blueberry

370 Vaccinium angustifolium; Javorek et al. 2002).

371

372 For community-level studies that use functional diversity approaches, our method could be used

373 to quantify hairiness for several body regions and weighted to give better representation of trait

374 diversity within the pollinator community. This is necessary where plant communities contain

375 diverse floral traits i.e. open-pollinated vs. closed-tubular flowers (Fontaine et al. 2006). Hairs on

376 different areas of the insect body are likely to vary in relative importance for pollen deposition

377 depending on trait matching (Bartomeus et al. 2016). Our method requires hairiness to be

378 measured at the individual-level (Figure S1), which makes it an ideal trait to use in new

379 functional diversity frameworks that use trait probabilistic densities rather than trait averages

380 (Carmona et al. 2016; Fontana et al. 2016). Combining predictive traits, such as pollinator

381 hairiness, with new methods that amalgamate intraspecific trait variation with multidimensional 
382 functional diversity, will greatly improve the explanatory power of trait-based pollination

383 studies.

384

385 One of the greatest constraints to advancing trait-based ecology is the time-demanding nature of 386 collecting trait data. This is because ecological communities typically contain many species,

387 which have multiple traits that need to be measured and replicated (Petchey \& Gaston 2006). To

388 improve the predictive power of trait-based ecology and streamline the data collection process

389 we must firstly identify traits that are strongly linked to ecosystem functions and secondly,

390 develop rigorous and time-efficient methodologies to measure traits at the individual level. We

391 achieve this by providing a method for quantifying a highly predictive trait at the individual-

392 level, in a time-efficient manner. Our method also complements other recently developed

393 predictive methods for estimating difficult-to-measure traits that are important for pollination

394 processes i.e. bee tongue length; Cariveau et al. (2016).

395

396 Predicating the functional importance of organisms is critical in a rapidly changing environment

397 where accelerating biodiversity loss threatens ecosystem functions (McGill et al. 2015). Our

398 novel method for measuring pollinator hairiness could be used in any studies that require

399 quantification of hairiness, such as understanding adhesion in insects (Bullock et al. 2008;

400 Clemente et al. 2010) or epizoochory (Albert et al. 2015; Sorensen 1986). It is also a much

401 needed addition to the pollination biologist's toolbox, and will progress the endeavour to

402 standardise trait-based approaches in pollination research. This is a crucial step towards

403 developing a strong mechanistic underpinning for trait-based pollination research. 


\section{Acknowledgements}

406 We thank David Seldon, Adrian Turner and Iain McDonald for assistance photographing insect

407 specimens, Anna Kokeny for help collecting specimens and Stephen Thorpe for assistance

408 identifying specimens. Patrick Garvey and Greg Holwell provided insightful comments on the

409 earlier manuscript. We also thank two anonymous reviewers for helpful and constructive

410 comments on the manuscript. We thank Sam Read, Brian Cutting, Heather McBrydie, Alex

411 Benoist, Rachel L'helgoualc'h and Simon Cornut for assistance in field work. Lastly, we thank

412 Estación Biológica de Doñana for hosting JS while developing the methodology for this paper.

\section{References}

415

416

417

418

419

420

421

422

423

424

425

426

427

428

429

430

431

432

433

434

435

436

437

438

439

440
Albert A, Auffret AG, Cosyns E, Cousins SAO, D'Hondt B, Eichberg C, Eycott AE, Heinken T, Hoffmann M, Jaroszewicz B, Malo JE, Mårell A, Mouissie M, Pakeman RJ, Picard M, Plue J, Poschlod P, Provoost S, Schulze KA, and Baltzinger C. 2015. Seed dispersal by ungulates as an ecological filter: A trait-based meta-analysis. Oikos 124:1109-1120. 10.1111/oik.02512

Bartomeus I, Bosch J, and Vilà M. 2008. High invasive pollen transfer, yet low deposition on native stigmas in a Carpobrotus-invaded community. Annals of botany 102:417-424.

Bartomeus I, Gravel D, Tylianakis JM, Aizen MA, Dickie IA, and Bernard-Verdier M. 2016. A common framework for identifying linkage rules across different types of interactions. Functional Ecology. 10.1111/1365-2435.12666

Bolnick DI, Amarasekare P, Araujo MS, Burger R, Levine JM, Novak M, Rudolf VH, Schreiber SJ, Urban MC, and Vasseur DA. 2011. Why intraspecific trait variation matters in community ecology. Trends in Ecology \& Evolution 26:183-192. 10.1016/j.tree.2011.01.009

Bullock JM, Drechsler P, and Federle W. 2008. Comparison of smooth and hairy attachment pads in insects: friction, adhesion and mechanisms for direction-dependence. The Journal of experimental biology 211:3333-3343.

Butterfield BJ, and Suding KN. 2013. Single-trait functional indices outperform multi-trait indices in linking environmental gradients and ecosystem services in a complex landscape. Journal of Ecology 101:9-17. 10.1111/1365-2745.12013

Cadotte MW, Carscadden K, and Mirotchnick N. 2011. Beyond species: functional diversity and the maintenance of ecological processes and services. Journal of Applied Ecology 48:1079-1087. 10.1111/j.1365-2664.2011.02048.x

Cane JH, Sampson BJ, and Miller SA. 2011. Pollination value of male bees: the specialist bee Peponapis pruinosa (Apidae) at summer squash (Cucurbita pepo). Environmental Entomology 40:614-620. 
441 Cariveau DP, Nayak GK, Bartomeus I, Zientek J, Ascher JS, Gibbs J, and Winfree R. 2016. The

442

443

444

445

446

447

448

449

450

451

452

453

454

455

456

457

458

459

460

461

462

463

464

465

466

467

468

469

470

471

472

473

474

475

476

477

478

479

480

481

482

483

484

485

486

allometry of bee proboscis length and its uses in ecology. PLoS ONE 11:e0151482.

10.1371/journal.pone.0151482

Carmona CP, de Bello F, Mason NWH, and Lepš J. 2016. Traits Without Borders: Integrating Functional Diversity Across Scales. Trends in Ecology \& Evolution.

10.1016/j.tree.2016.02.003

Clemente CJ, Bullock JM, Beale A, and Federle W. 2010. Evidence for self-cleaning in fluidbased smooth and hairy adhesive systems of insects. The Journal of experimental biology 213:635-642.

Craig JL, Stewart AM, Pomeroy N, Heath A, and Goodwin R. 1988. A review of kiwifruit pollination: where to next? New Zealand Journal of Experimental Agriculture 16:385399.

Crawley MJ. 2002. Statistical computing: an introduction to data analysis using S-Plus.-J. Statistical computing: an introduction to data analysis using S-Plus.

Dafni A. 2007. Pollination ecology. A practical approach. New York: Oxford University Press.

Devi I, Thakur BS, and Garg S. 2015. Floral morphology, pollen viability and pollinizer efficacy of kiwifruit. International Journal of Current Research and Academic Review 3:188-195.

Didham RK, Leather SR, and Basset Y. 2016. Circle the bandwagons - challenges mount against the theoretical foundations of applied functional trait and ecosystem service research. Insect Conservation and Diversity 9:1-3. 10.1111/icad.12150

Fontaine C, Dajoz I, Meriguet J, and Loreau M. 2006. Functional diversity of plant-pollinator interaction webs enhances the persistence of plant communities. PLoS Biology 4:01290135.

Fontana S, Petchey OL, Pomati F, and Sayer E. 2016. Individual-level trait diversity concepts and indices to comprehensively describe community change in multidimensional trait space. Functional Ecology 30:808-818. 10.1111/1365-2435.12551

Gagic V, Bartomeus I, Jonsson T, Taylor A, Winqvist C, Fischer C, Slade EMS-D, I., Emmerson M, Potts SG, Tscharntke T, Weisser W, and Bommarco R. 2015. Functional identity and diversity predict ecosystem functioning better than species-based indices. Proceedings of the Royal Society B: Biological Sciences 282:20142620. http://dx.doi.org/10.1098/rspb.2014.2620

Garibaldi LA, Bartomeus I, Bommarco R, Klein AM, Cunningham SA, Aizen MA, Boreux V, Garratt MPD, Carvalheiro LG, Kremen C, Morales CL, Schüepp C, Chacoff NP, Freitas BM, Gagic V, Holzschuh A, Klatt BK, Krewenka KM, Krishnan S, Mayfield MM, Motzke I, Otieno M, Petersen J, Potts SG, Ricketts TH, Rundlöf M, Sciligo A, Sinu PA, Steffan-Dewenter I, Taki H, Tscharntke T, Vergara CH, Viana BF, and Woyciechowski M. 2015. EDITOR'S CHOICE: REVIEW: Trait matching of flower visitors and crops predicts fruit set better than trait diversity. Journal of Applied Ecology 52:1436-1444. $10.1111 / 1365-2664.12530$

Gonzales RC, Woods RE, and Eddins SL. 2004. Digital image processing using MATLAB: Pearson Prentice Hall.

Goodwin RM, and Perry JH. 1992. Use of pollen traps to investigate the foraging behaviour of honey bee colonies in kiwifruit orchards. New Zealand Journal of Crop and Horticultural Science 20:23-26.

Herrera CM. 1987. Components of pollinator" quality": comparative analysis of a diverse insect assemblage. Oikos:79-90. 
487

488

489

490

491

492

493

494

495

496

497

498

499

500

501

502

503

504

505

506

507

508

509

510

511

512

513

514

515

516

517

518

519

520

521

522

523

524

525

526

527

528

529

530

531

Hillebrand H, and Matthiessen B. 2009. Biodiversity in a complex world: consolidation and progress in functional biodiversity research. Ecology Letters 12:1405-1419. 10.1111/j.1461-0248.2009.01388.x

Hoehn P, Tscharntke T, Tylianakis JM, and Steffan-Dewenter I. 2008. Functional group diversity of bee pollinators increases crop yield. Proceedings of the Royal Society B: Biological Sciences 275:2283-2291.

Holloway BA. 1976. Pollen-feeding in hover-flies (Diptera: Syrphidae). New Zealand Journal of Zoology 3:339-350. 10.1080/03014223.1976.9517924

Howlett BG, Walker MK, Rader R, Butler RC, Newstrom-Lloyd LE, and Teulon DAJ. 2011. Can insect body pollen counts be used to estimate pollen deposition on pak choi stigmas? New Zealand Plant Protection 64:25-31.

Javorek S, Mackenzie K, and Vander Kloet S. 2002. Comparative pollination effectiveness among bees (Hymenoptera: Apoidea) on lowbush blueberry (Ericaceae: Vaccinium angustifolium). Annals of the Entomological Society of America 95:345-351.

King C, Ballantyne G, and Willmer PG. 2013. Why flower visitation is a poor proxy for pollination: measuring single-visit pollen deposition, with implications for pollination networks and conservation. Methods in Ecology and Evolution 4:811-818. 10.1111/2041210X.12074

Klein A-M, Vaissiere BE, Cane JH, Steffan-Dewenter I, Cunningham SA, Kremen C, and Tscharntke T. 2007a. Importance of pollinators in changing landscapes for world crops. Proceedings of the Royal Society of London B: Biological Sciences 274:303-313.

Klein AM, Vaissiere BE, Cane JH, Steffan-Dewenter I, Cunningham SA, Kremen C, and Tscharntke T. 2007b. Importance of pollinators in changing landscapes for world crops. Proceedings of the Royal Society B: Biological Sciences 274:303-313.

Kremen C, Williams NM, Aizen MA, Gemmill-Herren B, LeBuhn G, Minckley R, Packer L, Potts SG, Roulston T, Steffan-Dewenter I, Vázquez DP, Winfree R, Adams L, Crone EE, Greenleaf SS, Keitt TH, Klein AM, Regetz J, and Ricketts TH. 2007. Pollination and other ecosystem services produced by mobile organisms: A conceptual framework for the effects of land-use change. Ecology Letters 10:299-314.

Kremen C, Williams NM, and Thorp RW. 2002. Crop pollination from native bees at risk from agricultural intensification. Proceedings of the National Academy of Sciences of the United States of America 99:16812-16816.

Larsen TH, Williams NM, and Kremen C. 2005. Extinction order and altered community structure rapidly disrupt ecosystem functioning. Ecology Letters 8:538-547. 10.1111/j.1461-0248.2005.00749.x

Lavorel S, Storkey J, Bardgett RD, de Bello F, and Berg MP. 2013. SPECIAL FEATURE: FUNCTIONAL DIVERSITY A novel framework for linking functional diversity of plants with other trophic levels for the quantification of ecosystem services. Journal of Vegetation Science 24:942-948.

Mayfield MM, Waser NM, and Price MV. 2001. Exploring the 'most effective pollinator principle' with complex flowers: bumblebees and Ipomopsis aggregata. Annals of botany 88:591-596.

McGill BJ, Dornelas M, Gotelli NJ, and Magurran AE. 2015. Fifteen forms of biodiversity trend in the Anthropocene. Trends in Ecology \& Evolution 30:104-113.

http://dx.doi.org/10.1016/j.tree.2014.11.006 
532 McGill BJ, Enquist BJ, Weiher E, and Westoby M. 2006. Rebuilding community ecology from

533

534

535

536

537

538

539

540

541

542

543

544

545

546

547

548

549

550

551

552

553

554

555

556

557

558

559

560

561

562

563

564

565

566

567

568

569

570

571

572

573

574

575

576

577

functional traits. Trends in Ecology \& Evolution 21:178-185.

http://dx.doi.org/10.1016/j.tree.2006.02.002

Naeem S, and Wright JP. 2003. Disentangling biodiversity effects on ecosystem functioning: deriving solutions to a seemingly insurmountable problem. Ecology Letters 6:567-579. 10.1046/j.1461-0248.2003.00471.x

Ne'eman G, Jürgens A, Newstrom-Lloyd L, Potts SG, and Dafni A. 2010. A framework for comparing pollinator performance: effectiveness and efficiency. Biological Reviews 85:435-451.10.1111/j.1469-185X.2009.00108.x

Ollerton J, Winfree R, and Tarrant S. 2011. How many flowering plants are pollinated by animals? Oikos 120:321-326.

Pasari JR, Levi T, Zavaleta ES, and Tilman D. 2013. Several scales of biodiversity affect ecosystem multifunctionality. Proceedings of the National Academy of Sciences 110:10219-10222.

Petchey OL, and Gaston KJ. 2006. Functional diversity: back to basics and looking forward. Ecology Letters 9:741-758. 10.1111/j.1461-0248.2006.00924.x

Potts SG, Dafni A, and Ne'eman G. 2001. Pollination of a core flowering shrub species in Mediterranean phrygana: variation in pollinator diversity, abundance and effectiveness in response to fire. Oikos 92:71-80.

R Development Core Team. 2014. R: A Language and Environment for Statistical Computing. (2014).

Rader R, Edwards W, Westcott DA, Cunningham SA, and Howlett BG. 2013. Diurnal effectiveness of pollination by bees and flies in agricultural Brassica rapa: Implications for ecosystem resilience. Basic and Applied Ecology 14:20-27. 10.1016/j.baae.2012.10.011

Rader R, Howlett BG, Cunningham SA, Westcott DA, Newstrom-Lloyd LE, Walker MK, Teulon DAJ, and Edwards W. 2009. Alternative pollinator taxa are equally efficient but not as effective as the honeybee in a mass flowering crop. Journal of Applied Ecology 46:1080-1087. 10.1111/j.1365-2664.2009.01700.x

Rathcke B. 1983. Competition and facilitation among plants for pollination. Pollination biology:305-329.

Roubik DW. 2000. Deceptive orchids with Meliponini as pollinators. Plant Systematics and Evolution 222:271-279. 10.1007/bf00984106

Shannon C. 1948. A mathematical theory of communication. Bell System Technical Journal 3:379-423. 10.1002/j.1538-7305.1948.tb01338.x.

Sorensen AE. 1986. Seed dispersal by adhesion. Annual Review of Ecology and Systematics:443463.

Thorp RW. 2000. The collection of pollen by bees. Pollen and pollination: Springer, 211-223.

Violle C, Enquist BJ, McGill BJ, Jiang L, Albert CH, Hulshof C, Jung V, and Messier J. 2012. The return of the variance: intraspecific variability in community ecology. Trends in Ecology \& Evolution 27:244-252. http://dx.doi.org/10.1016/j.tree.2011.11.014

Violle C, Navas M-L, Vile D, Kazakou E, Fortunel C, Hummel I, and Garnier E. 2007. Let the concept of trait be functional! Oikos 116:882-892. 10.1111/j.0030-1299.2007.15559.x

Walker B, Kinzig A, and Langridge J. 1999. Plant attribute diversity, resilience, and ecosystem function: the nature and significance of dominant and minor species. Ecosystems2: 95113. New York: Springer-Verlag. 


\section{Table $\mathbf{1}$ (on next page)}

Regression models examining the effect of entropy on SVD and pollen load

Top regression models examining the effect of insect body region entropy on single visit pollen deposition (SVD) for Brassica rapa and Actinidia deliciosa and pollen load for $B$. rapa. Models are presented in ascending order based on $\mathrm{AlC}_{\mathrm{C}}$ values. $\Delta i$ is the difference in the $\mathrm{AlC}_{\mathrm{C}}$ value of each model compared with the $\mathrm{AIC}_{\mathrm{C}}$ value for the top model. $w_{i}$ is the Akaike weight for each model and acc $w_{i}$ is the cumulative Akaike weight. Top models for each response variable are highlighted in bold. 


\begin{tabular}{|c|c|c|c|c|c|c|}
\hline $\begin{array}{l}\text { Response } \\
\text { variable }\end{array}$ & Model & $\operatorname{Adj} \mathbf{R}^{2}$ & $\mathrm{AIC}_{\mathrm{c}}$ & $\Delta i$ & $w_{i}$ & $\operatorname{acc} w_{i}$ \\
\hline \multirow{5}{*}{$\begin{array}{c}\text { SVD } \\
(\text { B. rapa })\end{array}$} & Face + Thorax dorsal & 0.98 & 88.29 & 0.00 & 0.82 & 0.82 \\
\hline & $\begin{array}{l}\text { Face }+ \text { Thorax dorsal }+ \\
\text { Abdomen ventral }\end{array}$ & 0.98 & 93.09 & 4.80 & 0.07 & 0.89 \\
\hline & $\begin{array}{l}\text { Face }+ \text { Head dorsal }+ \text { Thorax } \\
\text { dorsal }\end{array}$ & 0.98 & 93.81 & 5.52 & 0.05 & 0.94 \\
\hline & $\begin{array}{l}\text { Face }+ \text { Thorax ventral }+ \\
\text { Thorax dorsal }\end{array}$ & 0.97 & 96.59 & 8.29 & 0.01 & 0.96 \\
\hline & $\begin{array}{l}\text { Face }+ \text { Thorax dorsal }+ \text { Front } \\
\text { leg }\end{array}$ & 0.97 & 97.02 & 8.72 & 0.01 & 0.97 \\
\hline \multirow{5}{*}{$\begin{array}{l}\text { Pollen load } \\
(\text { B. rapa })\end{array}$} & Face & 0.81 & 168.47 & 0.00 & 0.64 & 0.64 \\
\hline & Abdomen dorsal & 0.73 & 171.59 & 3.12 & 0.13 & 0.78 \\
\hline & Face + Head dorsal & 0.83 & 173.59 & 5.12 & 0.05 & 0.83 \\
\hline & Face + Abdomen dorsal & 0.82 & 173.76 & 5.29 & 0.05 & 0.87 \\
\hline & Abdomen dorsal + Front leg & 0.80 & 174.86 & 6.39 & 0.03 & 0.90 \\
\hline \multirow{5}{*}{$\begin{array}{c}\text { SVD } \\
(\text { A. deliciosa })\end{array}$} & Face + Thorax ventral & 0.91 & 74.18 & 0.00 & 0.15 & 0.15 \\
\hline & Abdomen dorsal & 0.81 & 74.21 & 0.03 & 0.15 & 0.30 \\
\hline & Face & 0.80 & 74.35 & 0.17 & 0.14 & 0.45 \\
\hline & Head ventral & 0.79 & 74.84 & 0.66 & 0.11 & 0.56 \\
\hline & Abdomen ventral & 0.78 & 75.08 & 0.90 & 0.10 & 0.65 \\
\hline
\end{tabular}

1 


\section{Figure 1}

Entropy image of the face of a native New Zealand solitary bee Leioproctus paahaumaa.

Image of the face of a native New Zealand solitary bee Leioproctus paahaumaa (a) and the corresponding entropy image (b). Warmer colours on the entropy image represent higher entropy values (shown by the scale bar on the right). Black dots on the entropy image are near-round and small objects that have been removed from the analysis by the preprocessing function.

a

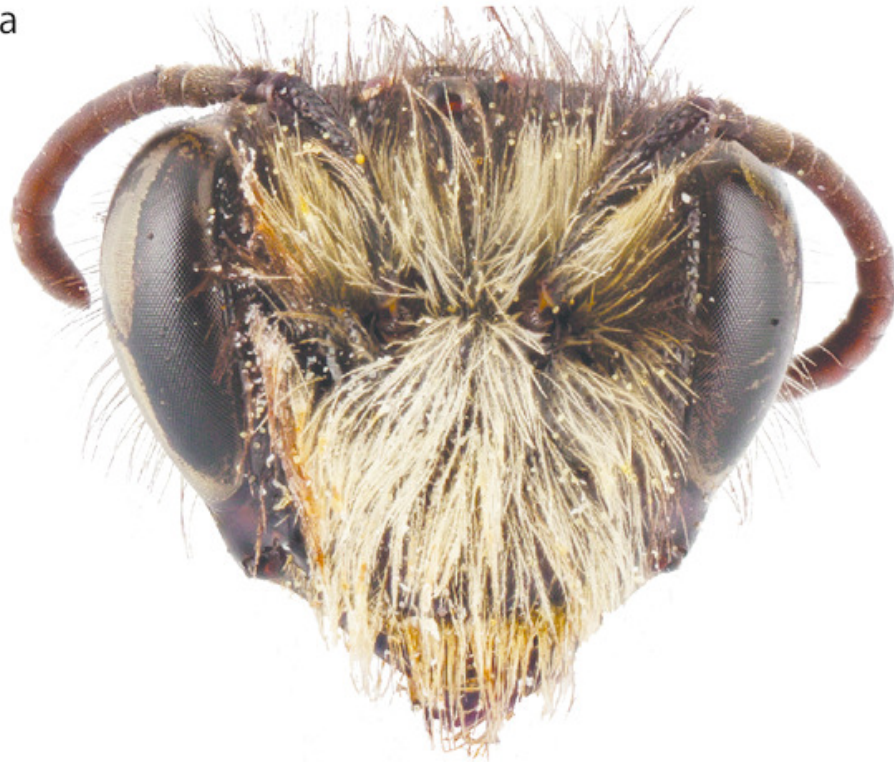

b

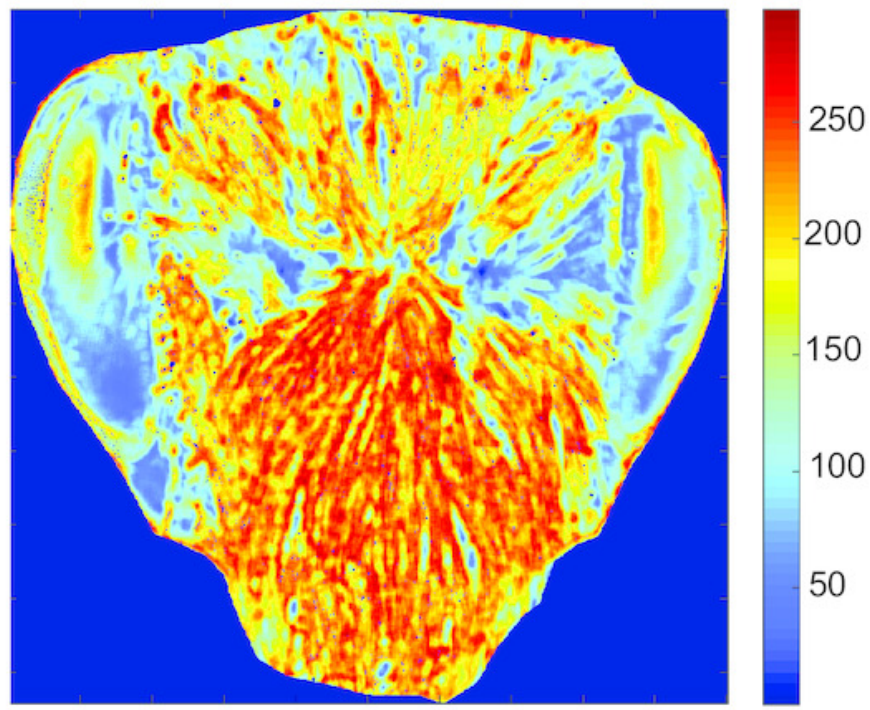


Figure 2

Relationships between mean entropy for each body region and mean single visit pollen deposition on Brassica rapa

Relationships between mean entropy for each body region and mean single visit pollen deposition (SVD) on Brassica rapa for 10 different insect pollinator species. Black lines are regressions for simple linear models.

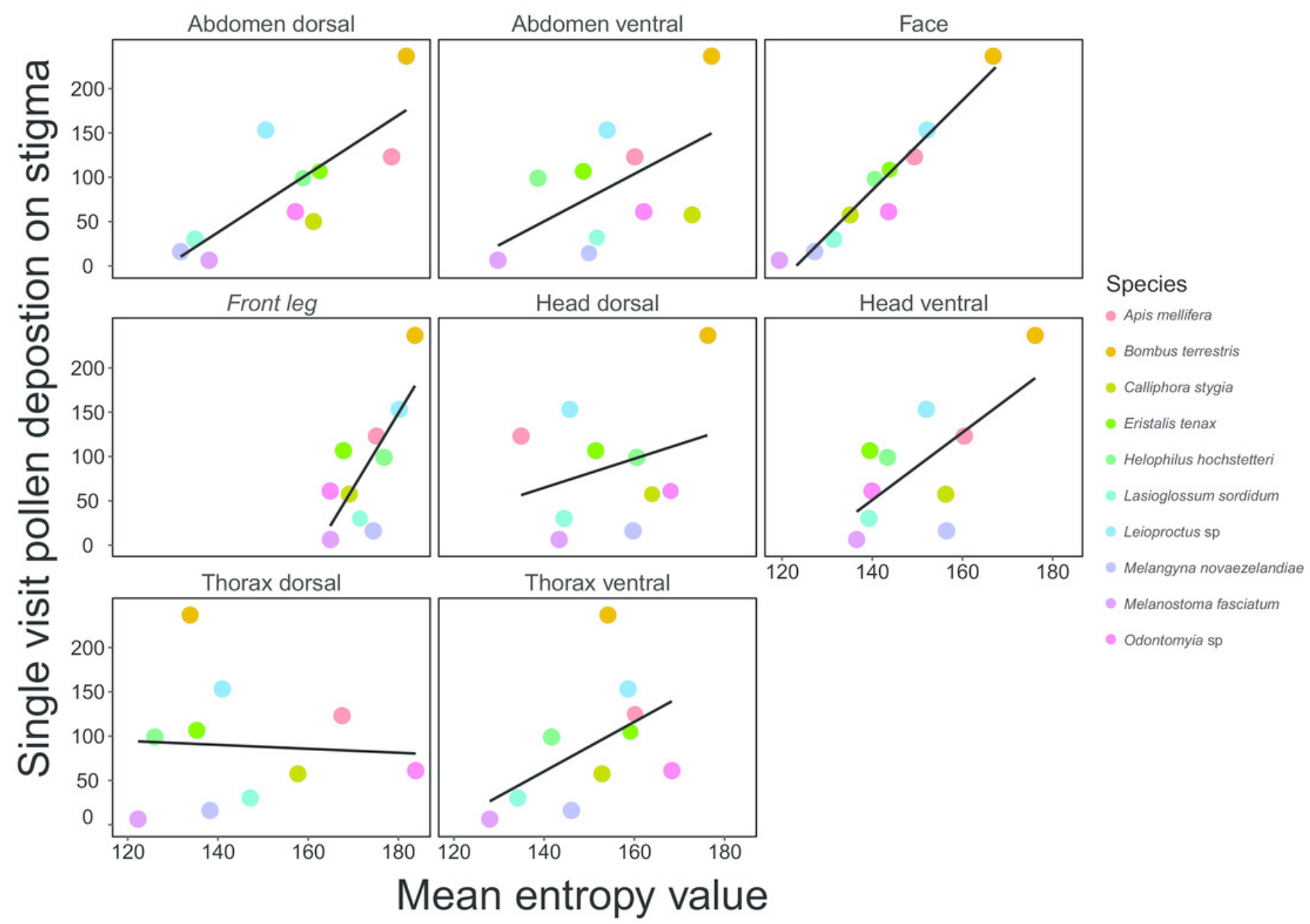


Figure 3

Relationships between mean entropy for each body region and mean single visit pollen deposition on Actinidia deliciosa

Relationships between mean entropy for each body region and mean single visit pollen deposition (SVD) on Actinidia deliciosa for 7 different insect pollinator species. Black lines are regressions for simple linear models.

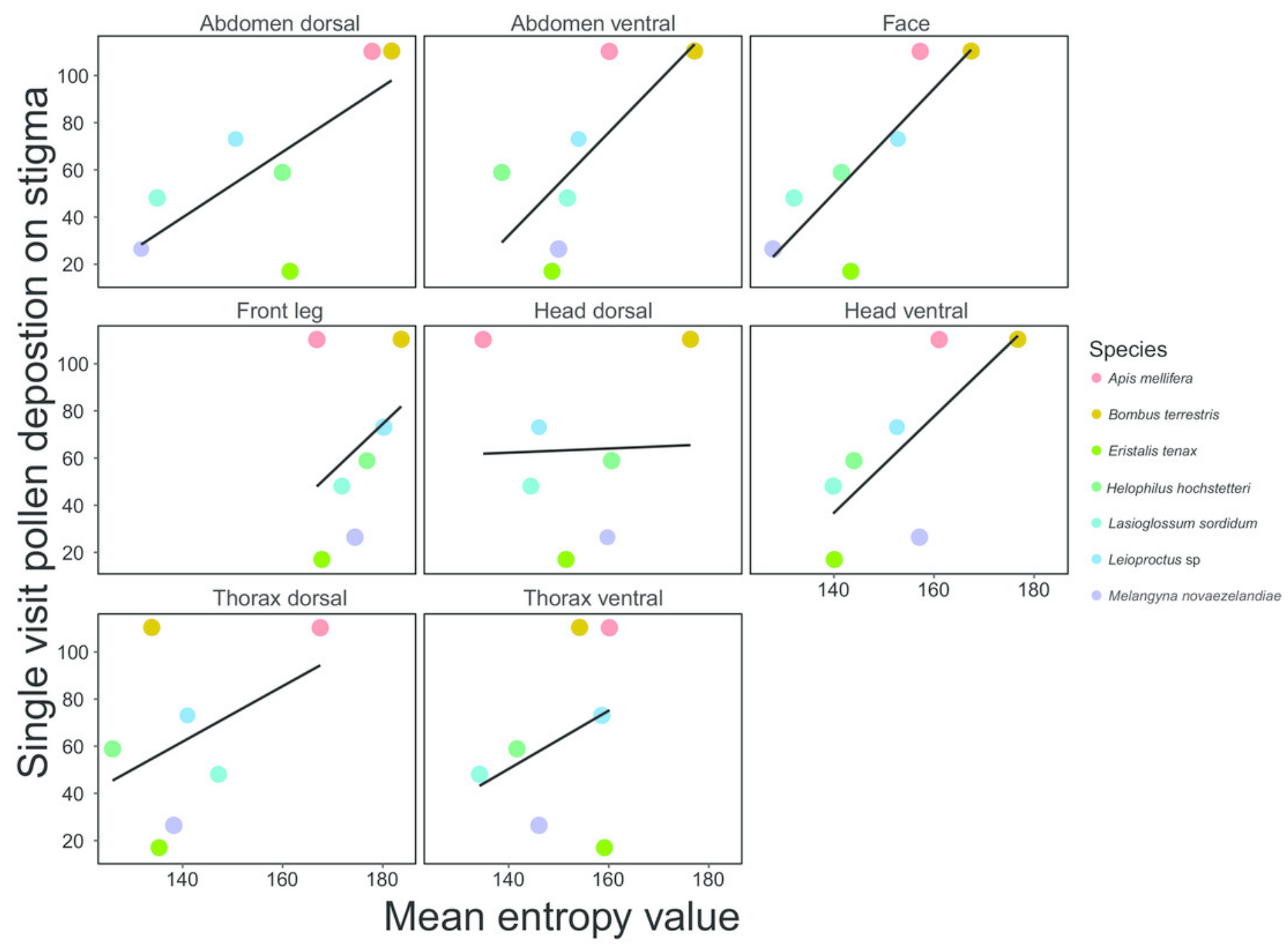


Figure 4

Relationship between entropy and Brassica rapa pollen load on insects

Relationships between mean entropy for each body region and the mean number of Brassica rapa pollen grains carried by 9 different insect pollinator species. Black lines are regressions for simple linear models.

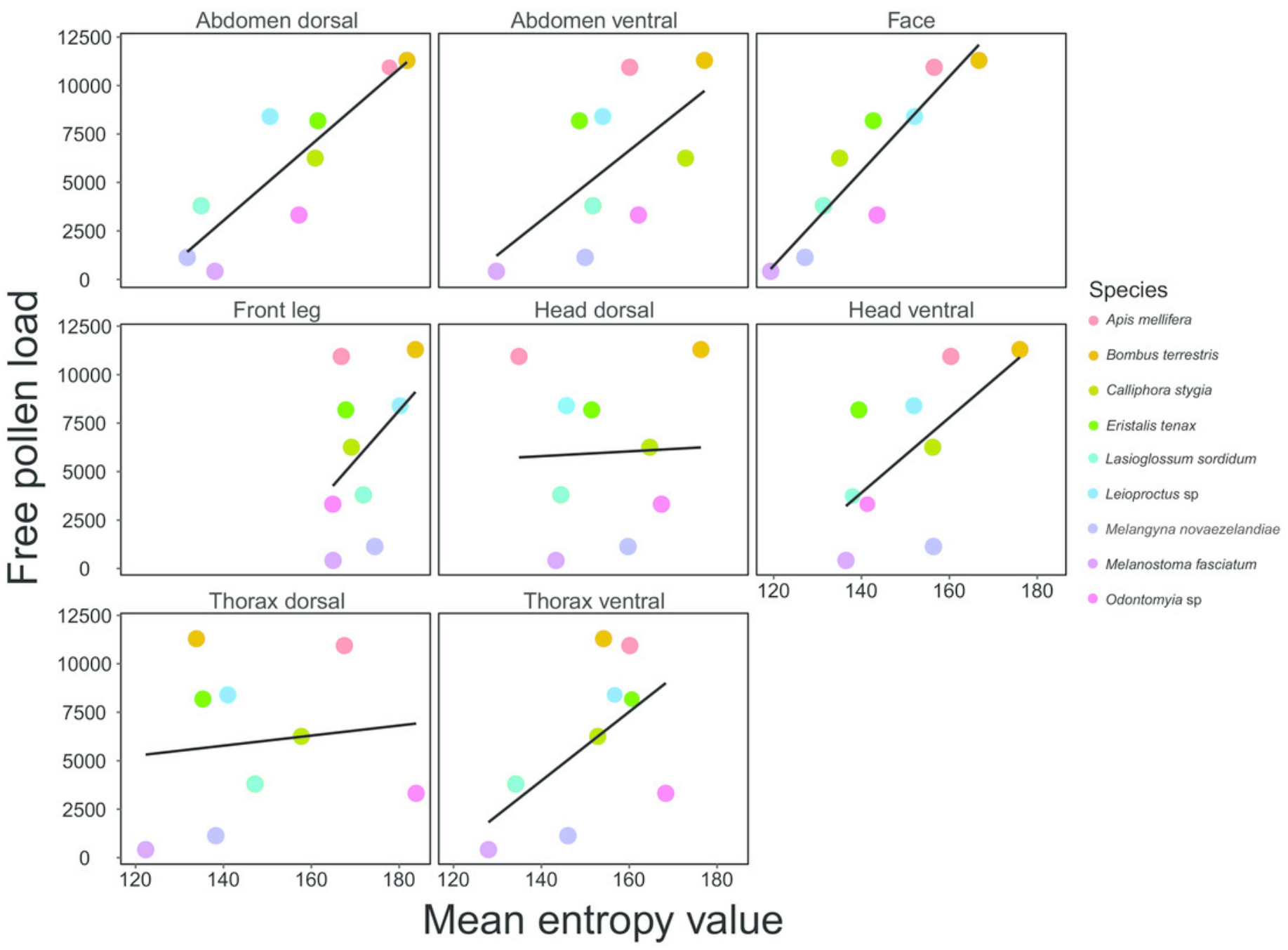

